\title{
Marcel CALVEZ (dir.), Santé et territoires. Des soins de proximité aux risques environnementaux
}

\section{Sylvain Bordiec}

\section{(2) OpenEdition}

Édition électronique

URL : http://journals.openedition.org/ress/3572

DOI : $10.4000 /$ ress. 3572

ISBN : $1663-4446$

ISSN : $1663-4446$

Éditeur

Librairie Droz

Édition imprimée

Date de publication : 30 novembre 2016

Pagination : 313-316

ISSN : 0048-8046

Référence électronique

Sylvain Bordiec, " Marcel CALVEZ (dir.), Santé et territoires. Des soins de proximité aux risques environnementaux», Revue européenne des sciences sociales [En ligne], 54-2 | 2016, mis en ligne le 22 août 2016, consulté le 25 septembre 2020. URL : http://journals.openedition.org/ress/3572 ; DOI : https://doi.org/10.4000/ress.3572

Ce document a été généré automatiquement le 25 septembre 2020.

(c) Librairie Droz 


\title{
Marcel CALVEZ (dir.), Santé et territoires. Des soins de proximité aux risques environnementaux
}

\author{
Sylvain Bordiec
}

\section{RÉFÉRENCE}

Marcel CALVEZ (dir.), 2016, Santé et territoires. Des soins de proximité aux risques environnementaux, Rennes, PUR, $204 \mathrm{p}$.

1 Cet ouvrage est issu des communications présentées lors d'un colloque organisé conjointement par la Maison des sciences de l'homme en Bretagne et la Maison des sciences de l'homme Ange-Guépin à Nantes et dédié à l'analyse des relations entre santé et territoires. Dirigé par Marcel Calvez, ce volume qui réunit des géographes, des sociologues, des juristes, des politistes, des politiques impliqués dans l'association Élus, Santé publique et Territoires et, enfin, un épidémiologiste, se propose, en privilégiant «des définitions larges» (p. 12) de ces notions de santé et de territoires, de construire une "posture distanciée à l'égard des approches en termes de dégradation pour chercher à qualifier différentes dimensions de ces relations » (p. 11). Comme l'explique le sociologue dans une introduction par ailleurs très instructive sur la construction historique, politique et juridique du «territoire socio-sanitaire » (p. 9), le fil conducteur de ce livre est le "rôle de l'environnement social dans la fabrication de la santé » (p. 14). Ici, dans la continuité des réflexions de Robert Putnam sur le capital social, l'environnement social désigne les «modalités d'organisation sociale comme la confiance, les normes et les réseaux qui peuvent améliorer l'efficacité de la société en facilitant les actions coordonnées entre individus »(ibid.). De fait, tout au long des 12 chapitres qui constituent cet ouvrage - et que nous nous proposons de passer en revue de manière non exhaustive -, il s'agit d'analyser « l'effet des territoires dans la fabrication de la santé » (p.15) en privilégiant « deux entrées complémentaires [...] du capital social» (ibid.). Faisant l'objet d'une première partie intitulée «Territoires de 
proximité et organisation des parcours de soins ", la première entrée interroge le "rôle de la puissance publique et des professions dans la construction d'un capital social identifiable par l'offre de soins dans un territoire » (ibid.). Ensuite, dans le cadre d'une deuxième partie intitulée "Territoires de vie et risques de santé ", la seconde entrée " aborde le territoire [...] investi de significations par ceux qui les utilisent à partir des menaces qu'il recèle » (p. 16). Pour Marcel Calvez, étant donné que « c'est [...] à l'échelle des territoires vécus par les populations que la question de la santé se pose, dans l'accès aux soins comme dans l'environnement de vie ou de travail » (p. 17), ces deux entrées sont par nature complémentaires.

Dans la première partie, à travers une mise en perspective de l'organisation de la santé en France avec le système québécois, Sébastien Fleuret (chapitre 1: "Questionner la territorialisation de la santé en France») soutient que le "millefeuille territorial» (p. 26) des services de santé en France est impuissant à constituer « un réseau territorialisé de première ligne » (p. 30): les territoires restent avant tout organisés « à travers l'application descendante de la politique nationale » (p. 33). Cette analyse fait écho aux considérations de François-Xavier Schweyer (chapitre 3: «Le territoire, un espace de réforme pour les soins de premier recours?») sur «l'usage ambigu de la notion de territoire» (p.45) dans la mise en œuvre, par une profession médicale peinant à trouver des candidats à la pratique dans «les zones fragiles» (p.47) et confrontée à la nécessité de la « coopération » (p. 53), d'une « organisation coordonnée des soins susceptible de répondre aux besoins de la population dans leur globalité » (p. 46). À partir du cas de la « prise en charge prénatale de proximité » (p. 71), laquelle serait dominée par le "principe de gestion de risques» (p. 74), Anne-Chantal Hardy (chapitre $5:$ « Penser les usages territoriaux de l'offre de santé. L'exemple de la prise en charge prénatale de proximité ») montre précisément que, plutôt que de créer les conditions d'une coordination génératrice d'une certaine égalité devant les risques prénataux, la territorialisation de la santé est au principe d'une «diversification de l'offre qui contribue à creuser les écarts de pratique» (p. 70). L'auteur rapporte ces inégalités à « un système de santé [qui] justifie l'offre libérale par le principe de "libre choix" des professionnels par les usagers" (p. 80). Comme le souligne la dernière contribution de la première partie (chapitre 7: «Appréhender les constructions territoriales des inégalités d'accès au dépistage du cancer du sein à Gonesse (Val d'Oise) : diagnostic territorial et aide à la décision »), rédigée par un collectif d'auteurs, ce type d'inégalités est indissociablement social et spatial et opère aussi bien entre les collectivités qu'à l'intérieur de ces dernières, notamment à l'échelle des quartiers. Ces dynamiques résultent "d'un cumul d'éléments localisés tels que le niveau socioéconomique [...], le rôle des acteurs locaux [...] et la création de conditions d'une prise de conscience et d'émulation collective" (p.122). Celles-ci posent "une question d'équité territoriale » (ibid.) à laquelle le «diagnostic territorial » peut apporter, en constituant un "outil mobilisateur ou remobilisateur des acteurs» (p.123), des éléments de compréhension et de réponse. Tout en déplaçant le regard sur les liens entre santé et environnement, la réflexion développée dans la seconde partie reste attentive à cette thématique des inégalités et aux moyens à mettre en œuvre pour les résorber.

3 Selon Isabelle Roussel (chapitre 8: "Territorialisation des risques sanitaires et inégalités environnementales »), la compréhension des inégalités environnementales nécessite de croiser «la cartographie du risque sanitaire lié à l'exposition d'un 
territoire à une source de pollution» (p. 140) avec la perception des habitants. La géographe va plus loin en soutenant que « c'est le territoire tel qu'il est vécu et non pas statistiquement objectivé qui doit piloter les décisions politiques » (p. 138). Ce territoire vécu par les populations est indissociable du travail, lequel peut être à l'origine de cancers d'origine professionnelle. C'est l'objet de la réflexion menée par Véronique Daubas-Letourneux et Christophe Coutanceau à partir du cas du port de Nantes (chapitre 10: «Identifier les expositions sur le Grand Port maritime de Nantes/SaintNazaire. Le projet ESCALES). La recherche-action à laquelle ces auteurs contribuent vise "à intégrer la dimension temporelle et cumulative de l'histoire d'exposition inscrite dans les parcours professionnels » (p. 162). Cette démarche doit servir à la construction d'un réseau de " partage des connaissances sur les lieux (quai, navire), marchandises, procédés qui provoquent des maladies pour agir en prévention» (p. 168). Comme le montre Marcel Calvez (chapitre 11: «Qualité de vie, maladies et territoires. Ce que disent les plaintes en santé environnementale»), le territoire vécu est également saisissable à travers les "plaintes en santé environnementale » (p. 171). La durée et l'ampleur des mobilisations, de même que la portée des initiatives, sont inégales selon les territoires. D'après l'auteur, il y a « des probabilités plus grandes de voir émerger des questions de santé environnementale concernant le milieu de vie dans des territoires où des réseaux existent [...], en particulier dans les territoires ruraux, plus que dans des territoires urbains, où les acteurs sont plus atomisés » (p. 182). Véronique Van Tilbeurgh et Sylvie Ollitrault (chapitre 12: «Plaintes de santé et attribution de confiance dans des réseaux territorialisés ») soulignent enfin l'« incertitude » (p. 188) qui s'empare des personnes mobilisées contre ce qu'elles perçoivent comme un nouveau risque sanitaire dans leur environnement. Leur étude de deux dispositifs sociotechniques - des parcs éoliens dans le Finistère et des antennes-relais à Rennes montre comment chaque plainte et chaque mobilisation s'organisent dans un cadre associatif et s'énoncent, se légitiment ou se délégitiment dans des relations de confiance et/ou de défiance avec les pouvoirs locaux. D'après les deux auteurs, « dans le contexte environnemental et politique actuel, les risques pour la santé sont alors un argument ultime pour faire valoir leurs enjeux de positionnement social dans le territoire qu'ils habitent $»$ (p. 198).

4 L'ouvrage convainc pleinement de la nécessité qu'il y a, pour comprendre les relations entre santé et territoires, de multiplier les niveaux d'analyse - géographique, sociologique, politique et juridique mais aussi épidémiologique - des interactions entre population et espace, monde professionnel et monde privé, inscriptions territoriales de l'existence et opportunités de mobilisation afin de cerner un problème de santé et/ou environnemental. Une des forces du livre réside également dans sa mise au jour de l'articulation entre le territoire comme lieu d'organisation des soins et de rapports pratiques au secteur médical et à l'environnement, d'un côté, et, de l'autre, le territoire comme générateur de représentations sur la manière dont celui-ci expose ses habitants à des risques sanitaires ou, au contraire, les en protège. Ces représentations jouent un rôle crucial dans les possibilités - et les impossibilités - d'engagement local dans un enjeu sanitaire et environnemental. En définitive, l'attention largement prêtée ici aux dispositifs officiels de mise en œuvre d'une gestion territorialisée de la santé, aux discours des plaintes et aux mises en forme des mobilisations se fait au détriment d'un intérêt plus poussé pour les ressorts de classe des relations pratiques et symboliques à ces enjeux sanitaires et environnementaux. Certes, le projet de connaissance annoncé eût été encore plus abouti en accordant plus d'importance aux propriétés sociales de 
ces professionnels, militants et habitants dans les territoires. La lecture de ce livre n'en reste pas moins essentielle pour quiconque veut comprendre comment, dans les sphères académique, militante, administrative, juridique et médicale, les influences réciproques entre santé et territoires sont pensées et traitées.

\section{AUTEURS}

\section{SYLVAIN BORDIEC}

Université de Bordeaux - Collège Sciences de l'homme - Faculté des Staps 